\title{
A Comparison Between Nonlinear Force-Free Field and Potential Field Models Using Full-Disk SDO/HMI Magnetogram
}

\author{
Tilaye Tadesse ${ }^{1}$, T. Wiegelmann ${ }^{3}$, \\ B. Inhester ${ }^{3}$, K. Olson ${ }^{1}$, P. J. MacNeice ${ }^{4}$, \\ A. Pevtsov ${ }^{5}$
}

(C) Springer $\bullet \bullet \bullet \bullet$

\begin{abstract}
Measurements of magnetic fields and electric currents in the preeruptive corona are crucial to study solar eruptive phenomena, like flare and coronal mass ejections(CMEs). However, spectro-polarimetric measurements of certain photospheric lines permit a determination of the vector magnetic field at the photosphere. Thus, substantial collection of magnetograms relate to the photospheric surface field only. Therefore, there is considerable interest in accurate modeling of the solar coronal magnetic field using photospheric vector magnetograms as boundary data. This numerical modeling is carried out by applying state-of-the-art nonlinear force-free field (NLFFF) reconstruction. Cartesian nonlinear force-free field (NLFFF) codes are not well suited for larger domains, since the spherical nature of the solar surface cannot be neglected when the field of view is large. One of the most significant results of Solar Dynamic Observatory (SDO) mission to date has been repeated observations of large, almost global scale events in which large scale connection between active regions may play fundamental role. Therefore, it appears prudent to implement a NLFFF procedure in spherical geometry for use when large scale boundary data are available, such as from the Helioseismic and Magnetic Imager (HMI) on board SDO. In this work, we model the coronal magnetic field above multiple active regions with the help of a potential field and a NLFFF extrapolation codes in a full-disk using HMI data as a boundary conditions. We compare projections of the resulting magnetic field lines solutions with full-disk coronal images from the Atmospheric Imaging Assembly (SDO/AIA) for both models. This study
\end{abstract}

\footnotetext{
1 Department of Physics, Drexel University, Philadelphia, PA 19104-2875, U.S.A, email: tasfaw@einstein.physics.drexel.edu email:

Kevin.M.Olson@drexel.edu

3 Max Planck Institut für Sonnensystemforschung,

Max-Planck Str. 2, D-37191 Katlenburg-Lindau, Germany,

email: wiegelmann@mps.mpg.de, email: inhester@mps.mpg.de

4 NASA, Goddard Space Flight Center, Code 674, Greenbelt,

MD 20771, U.S.A. email: peter.j.macneice@nasa.gov

5 National Solar Observatory, Sunspot, NM 88349, U.S.A.

email: apevtsov@nso.edu
} 
has found that the NLFFF model reconstructs the magnetic configuration better than the potential field model. We have concluded that much of trans-equatorial loops connecting the two solar hemispheres are current-free.

Keywords: Active Regions, Magnetic Fields; Active Regions, Models; Magnetic fields, Corona; Magnetic fields, Photosphere; Magnetic fields, Models

\section{Introduction}

Magnetic fields are believed to play a dominant role for active phenomena carried out in the solar corona. The study of solar eruptive phenomena requires that we understand how magnetic energy is stored in the pre-eruptive corona. One of the key questions is: What is the three dimensional (3D) structure of magnetic fields and electric currents in the pre-eruptive corona, and how much free energy is stored in the field? (Schrijver and Title, 2005, Jiang and Feng, 2012). To answer this question we must measure the magnetic field in the coronal volume. However, spectro-polarimetric measurements of certain photospheric lines permit a determination of the vector magnetic field at the photosphere. Thus, substantial collection of magnetograms relate to the photospheric surface field only. Although measurement of magnetic fields in the chromosphere and the corona has considerably improved in recent decades (Lin, Penn, and Tomczyk, 2000; Liu and Lin, 2008), further developments are needed before accurate data are routinely available. The problem of measuring the coronal field and its embedded electrical currents thus leads us to use numerical modeling to infer the field strength in the higher layers of the solar atmosphere from the measured photospheric field. Due to the low value of the plasma $\beta$ (the ratio of gas pressure to magnetic pressure), the solar corona is magnetically dominated (Gary, 2001). To describe the equilibrium structure of the static coronal magnetic field when non-magnetic forces are negligible, the force-free assumption is appropriate:

$$
\begin{gathered}
(\nabla \times \mathbf{B}) \times \mathbf{B}=0 \\
\nabla \cdot \mathbf{B}=0
\end{gathered}
$$

subject to the boundary condition

$$
\mathbf{B}=\mathbf{B}_{\mathrm{obs}} \quad \text { on photosphere }
$$

where $\mathbf{B}$ is the magnetic field and $\mathbf{B}_{\mathrm{obs}}$ is measured vector field on the photosphere. Equation (1) states that the Lorentz force vanishes (as a consequence of $\mathbf{J} \| \mathbf{B}$, where $\mathbf{J}$ is the electric current density) and Equation (2) describes the absence of magnetic monopoles.

As an alternative to real measurements, force-free extrapolation of photosphericmagnetic fields is currently being used as a the primary tool for the modeling of coronal magnetic fields (Inhester and Wiegelmann, 2006, Valori, Kliem, and Keppens, 2005, Wiegelmann, 2004; Wheatland, 2004; Wheatland and Régnier, 2009; Tadesse, Wiegelmann, and Inhester, 2009, Wheatland and 
Leka, 2011; Amari and Aly, 2010; Wiegelmann, T. and Thalmann, J. K. and Inhester, B. and Tadesse, T. et al., 2012 ; Jiang and Feng, 2012, Jiang, Feng, and Xiang, 2012 Aschwanden and Malanushenko, 2012 ; Wiegelmann and Sakurai, 2012).

Cartesian nonlinear force-free field (NLFFF) codes are not well suited for larger domains, since the spherical nature of the solar surface cannot be neglected when the field of view is large (Wiegelmann, 2007: Tadesse, Wiegelmann, and Inhester, 2009: Tadesse et al., 2012a: Guo et al., 2012). One of the most significant results of Solar Dynamic Observatory (SDO) mission to date has been repeated observations of large, almost global scale events in which large scale connection between active regions may play fundamental role. Therefore, it appears prudent to implement a NLFFF procedure in spherical geometry for use when large scale boundary data are available, such as from the Helioseismic and Magnetic Imager (HMI) on board SDO. DeRosa, M. L. and Schrijver, C. J. and Barnes, G. et al. (2009) has studied that different NLFFF models have markedly different field line configurations and provided widely varying estimates of the magnetic free energy in the coronal volume. The main reasons for that problem are (1) the forces acting on the field within the photosphere, (2) the uncertainties on vectorfield measurements, particularly on the transverse component, and (3) the large domain that needs to be modeled to capture the connections of an active region to its surroundings (Wiegelmann, 2007, Tadesse et al., 2011; Tadesse et al., 2012b; Tadesse et al., 2012c). In this study, we have considered those three points explicitly into account for modeling coronal magnetic field in a full-disk.

In this work, we apply our spherical NLFFF procedure to a group of active regions observed on November 092011 around 17:45UT by SDO/HMI. During this observation, there were four active regions (ARs 11338, 11339, 11341 and 11342) along with other smaller sunspots spreading on the disk. We compare the extrapolated (both potential and NLFFF) magnetic loops with extreme ultraviolet (EUV) observations by the Atmospheric Imaging Assembly (AIA) (Lemen, J. R. and Title, A. M. and Akin, D. J. et al., 2012) on board SDO. During comparison, we check whether the NLFFF model reconstructs the magnetic configuration better than the potential field model. This comparison can be used to evaluate how well the model field lines approximate the observed coronal loops.

\section{Instrumentation and data set}

The Helioseismic and Magnetic Imager (HMI) (Schou, J. and Scherrer, P. H. and Bush, R. I. et al., 2012) is part of the Solar Dynamics Observatory (SDO) and observes the full Sun at six wavelengths and full Stokes profile in the Fe slowromancapi@617.3 nm spectral line. It consists of a refracting telescope, a polarization selector, an image stabilization system, a narrow band tunable filter and two 4096 pixel CCD cameras with mechanical shutters and control electronics.

The transverse components of vector magnetic fields suffer from the so-called $180^{\circ}$ ambiguity. The $180^{\circ}$ ambiguity for the HMI data in this study has been 
resolved by an improved version of the minimum energy method (Metcalf, 1994; Metcalf, T. R. and Leka, K. D. and Barnes, G. et al., 2006 Leka, Barnes, and Crouch, 2009). As described in Leka, Barnes, and Crouch (2009), in weak-field areas, the minimization may not return a good solution due to large noise. The noise level is $\approx 10 \mathrm{G}$ and $\approx 100 \mathrm{G}$ for the longitudinal and transverse magnetic field, respectively. Therefore, in order to get a spatially smooth solution in weakfield areas, we divide the magnetic field into two regions, i.e., strong-field region and weak-field region, which is defined to be where the field strength is below $200 \mathrm{G}$ at the disk center, and $400 \mathrm{G}$ on the limb. The values vary linearly with distance from the center to the limb. The ambiguity solution in the strong-field area is derived by the annealing in the minimization method and released in the data series "hmi.B_720s_e15w1332_cutout". Magnetic field azimuths in the weak-field area are finally determined by the potential-field acute-angle method after the annealing. This is different from the original minimum energy method, which uses a neighboring-pixel acute-angle algorithm to revisit the weak field area. Figure 3 a) shows the vector magnetic fields observed by HMI on November 092011 around 17:45UT.

\section{Optimization principle and preprocessing of HMI data}

Molodensky (1969) and Aly (1989) pointed out that vector magnetic fields on a closed surface that fully encloses any force-free domain have to satisfy the force-free and torque-free conditions. To serve as suitable lower boundary condition for a force-free modeling, on average a net tangential force acting on the boundary and shear stresses along axes lying on the boundary have to reduce to zero (Molodensky, 1969, Aly, 1989, Wiegelmann, Inhester, and Sakurai, 2006: Tadesse, Wiegelmann, and Inhester, 2009). Preprocessing method as implemented in Wiegelmann, Inhester, and Sakurai (2006) has to be used to fulfill those conditions. The preprocessing scheme of Tadesse, Wiegelmann, and Inhester (2009) involves minimizing a two-dimensional functional of quadratic form in spherical geometry similar to

$$
\begin{gathered}
\mathbf{B}=\operatorname{argmin}\left(L_{p}\right), \\
L_{p}=\mu_{1} L_{1}+\mu_{2} L_{2}+\mu_{3} L_{3}+\mu_{4} L_{4},
\end{gathered}
$$

where $\mathbf{B}$ is the preprocessed surface magnetic field from the input observed field $\mathbf{B}_{o b s}$. Each of the constraints $L_{n}$ is weighted by an as yet undetermined factor $\mu_{n}$. The first term $(n=1)$ corresponds to the force-balance condition, the next $(n=2)$ to the torque-free condition, and the last term $(n=4)$ controls the smoothing. The explicit form of $L_{1}, L_{2}$, and $L_{4}$ can be found in Tadesse, Wiegelmann, and Inhester (2009). The term $(n=3)$ controls the difference between measured and preprocessed vector fields. After preprocessing the HMI data, we solve the force-free equations (1) and (2) using optimization principle (Wheatland, Sturrock, and Roumeliotis, 2000 Wiegelmann, 2004) in spherical geometry (Wiegelmann, 2007) Tadesse, Wiegelmann, and Inhester, 2009: Tadesse et al., 2012c). 


\section{Potential field model}

The simplest way to model the coronal field is to assume that it is potential, i.e. that it carries no electric current. Solutions for this model in plane geometry have been obtained by Schmidt (1964), for the case where the vertical component of the field is specified at the photospheric boundary. Potential field model has now led to an almost routine type reconstruction, used for observational purposes (Sakurai, 1989), but also for building initial conditions for dynamical MHD numerical simulations (Amari et al., 1996). This assumption has proven to be adequate for many quiescent, old active regions and even for the non-eruptive global coronal-heliospheric interface (Wang and Sheeley, 1990, Schrijver and De Rosa, 2003).

Studies of the coupling of the coronal field into the heliosphere suggest that the global coronal magnetic field is often largely potential. For the practical calculation of the global field, the so-called source-surface model has been introduced (Schatten, Wilcox, and Ness, 1969), in which the influence of the solar wind is artificially taken account of by the requirement that the field be radial at some exterior spherical (source) surface typically at $R_{s}=2.5 R_{\odot}$ from the suns center. The potential-field source surface (PFSS) model, uses this concept to extrapolate the line-of-sight surface magnetic field through the corona with the boundary assumed to be at the source surface.

The potential field approximation has often been used to deduce the magnetic structure of the solar corona from measurements of the photospheric field. It obeys the equation $\nabla \times \mathbf{B}=0$, so that the magnetic field can be expressed as the gradient of a scalar potential, i.e. $\mathbf{B}=-\nabla \Phi$. Since the magnetic field is divergence-free $(\nabla \cdot \mathbf{B}=0)$, the scalar potential obeys the Laplace equation $\nabla^{2} \Phi=0$. Together with the photospheric boundary condition, which is traditionally provided by a map of the radial magnetic field, the Laplace equation can be solved in the spherical coordinate system $(r, \theta, \phi)$, where $\theta$ stands for colatitude. Using the radial magnetic field observed at $R_{\odot}$ and the source surface assumption at $R_{s}$ as the boundary condition, together with the spherical harmonic expansion in the domain $R_{\odot}<r<R_{s}$, we finally obtain the potential field model.

\section{Results}

In this study, we have used potential field model and our spherical NLFFF optimization procedure to a group of active regions observed on November 09 2011 around 17:45UT by SDO/HMI instrument. During this observation there were four active regions (ARs 11338, 11339, 11341 and 11342, see Fig. 2a) along with other smaller sunspots spreading on the disk. In order to accommodate the connectivity between those ARs and their surroundings, and to study transequatorial loops (loops connecting north and south hemisphere), we adopt a non uniform spherical grid $r, \theta, \phi$ with $n_{r}=225, n_{\theta}=375, n_{\phi}=425$ grid points in the direction of radius, latitude, and longitude, respectively, with the field of view of $\left[r_{\min }=1 R_{\odot}: r_{\max }=2 R_{\odot}\right] \times\left[\theta_{\min }=-50^{\circ}: \theta_{\max }=50^{\circ}\right] \times\left[\phi_{\min }=90^{\circ}:\right.$ 
$\phi_{\max }=270^{\circ}$. The main purpose of this work is to study the magnetic and electric current connectivities of the northern and southern solar hemispheres and to compare which of the two models (potential or NLFFF model) is best suited to fully describe those connectivities in full-disk environment.

We use our preprocessing routine in spherical geometry to derive suitable boundary conditions for force-free modeling from the measured photospheric data (Wiegelmann, Inhester, and Sakurai, 2006, Tadesse, Wiegelmann, and Inhester, 2009). We used a standard preprocessing parameter set $\mu_{1}=\mu_{2}=1$, $\mu_{3}=0.001$ and $\mu_{4}=0.05$, which are similar to the values calculated from vector data used in previous studies (Wiegelmann, T. and Thalmann, J. K. and Inhester, B. and Tadesse, T. et al., 2012; Tadesse et al., 2012c) for HMI data. The preprocessing influences the structure of the magnetic vector data. It does not only smooths $\mathbf{B}_{t}$ (transverse field) but also alters its values in order to reduce the net force and torque. The change in $\mathbf{B}_{t}$ is more pronounced than the radial component $\mathbf{B}_{r}$ (radial field) since $\mathbf{B}_{t}$ is measured with lower accuracy than the longitudinal magnetic field.

For computing the potential field, we use the preprocessed radial component $\mathbf{B}_{r}$ of the HMI-data using spherical harmonic expansion. This potential field solution has been used to initialize our NLFFF code. For nonlinear force-free fields we minimize the functional Eq. (??). We implement the new term $L_{\text {photo }}$ in Eq. (??) to work with boundary data of different noise levels and qualities (Wiegelmann and Inhester, 2010; Tadesse et al., 2011). For those pixels, for which $\mathbf{B}_{\text {obs }}$ was successfully inverted, we allow deviations between the model field $\mathbf{B}$ and the input fields observed $\mathbf{B}_{\text {obs }}$ surface field using Eq. (??), so that the model field can be iterated closer to a force-free solution even if the observations are inconsistent. In order to control the speed with which the lower boundary is injected during the NLFFF extrapolation, we have used the Langrangian multiplier of $\nu=0.001$ as suggested by Tadesse et al. (2012c). For more details of the method used in this work we direct the readers to the study by Tadesse et al. (2011).

We plot the surface potential field and NLFFF solutions in Figure 1 for selected region excluding the polar regions. In this figure, one can see that there are substantial differences between the surface radial and transverse field components of the two models. To quantify the degree of disagreement between the respective vector components of potential and NLFFF model solutions on the bottom surface, we calculate their pixel-wise correlations. The correlation were calculated(Schrijver, C. J. and Derosa, M. L. and Metcalf, T. R. et al., 2006: Metcalf, T. R. and De Rosa, M. L. and Schrijver, C. J. et al., 2008) from

$$
C_{\mathrm{vec}}=\frac{\sum_{i} \mathbf{v}_{i} \cdot \mathbf{u}_{i}}{\left(\sum_{i}\left|\mathbf{v}_{i}\right|^{2} \sum_{i}\left|\mathbf{u}_{i}\right|^{2}\right)^{1 / 2}}
$$

where $\mathbf{v}_{i}$ and $\mathbf{u}_{i}$ are the vectors at each grid point $i$ on the bottom surface. If the vector fields are identical, then $C_{v e c}=1$; if $\mathbf{v}_{i} \perp \mathbf{u}_{i}$, then $C_{v e c}=0$. Table 1 shows the correlation $\left(C_{v e c}\right)$ of the $2 \mathrm{D}$ surface magnetic field vectors of potential and NLFFF models for the radial and transverse components. The vector correlation between $\mathbf{B}_{t}$ of the potential and NLFFF surface vector maps 


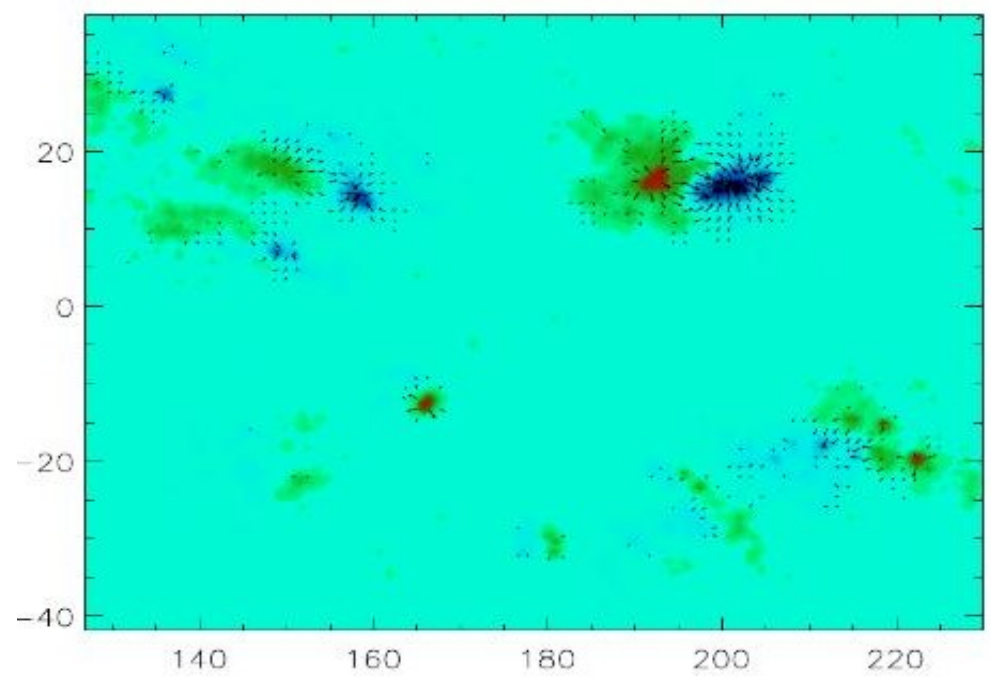

(a) Surface potential field solution

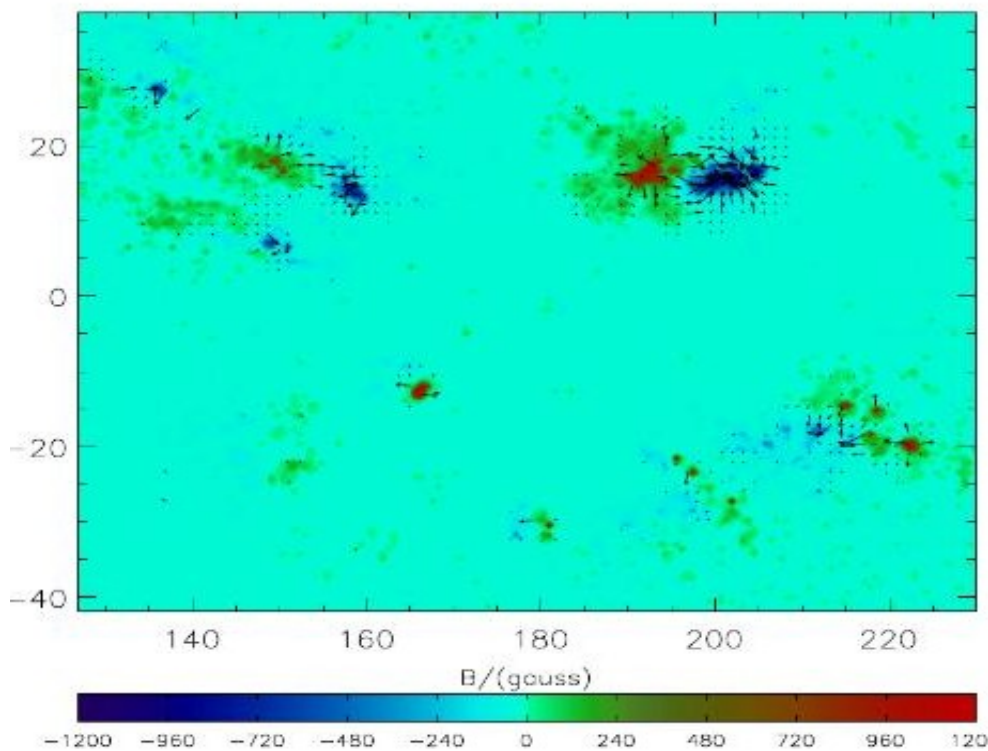

(b) Surface NLFF field solution

Figure 1. Magnetic vector maps of HMI data on part of the lower boundary. The color coding shows $B_{r}$ on the photosphere and the black arrow indicates the transverse components of the field. The vertical and horizontal axes show latitude, $\theta$ and longitude, $\phi$ on the photosphere respectively. 
Table 1. The correlations between the components of surface fields from potential and NLFFF models.

\begin{tabular}{ccc}
\hline & & \\
\hline $\mathrm{v}$ & $\mathrm{u}$ & $C_{\mathrm{vec}}$ \\
\hline$\left(\mathbf{B}_{\text {pot }}\right)_{r}$ & $\left(\mathbf{B}_{N L F F F}\right)_{r}$ & 0.937 \\
$\left(\mathbf{B}_{\text {pot }}\right)_{t}$ & $\left(\mathbf{B}_{N L F F F}\right)_{t}$ & 0.813 \\
\hline
\end{tabular}

is much more less than that of respective $\mathbf{B}_{r}$ components. This is due to the fact that the transverse components $\left(\mathbf{B}_{t}\right)$ of the potential field model are computed using spherical harmonics expansion from the radial components of the magnetic field used as a boundary condition for NLFFF model. The average vector correlations between the radial and transverse components of the two models in Table1 indicates that the NLFFF solutions are somewhat far from potential as they carry electric currents. In order to compare our reconstructions with observation, we plot the selected fieldlines of the potential and NLFFF models in Figure 2 and d. We overlay the field lines with AIA $193 \AA$ image. For observation time in Figure $2 \mathrm{r}$ and $\mathrm{d}$, the field lines of the potential and NLFFF models are reconstructed from the same footpoints. The potential field lines in Figure 2(c) have an obvious deviation from the observed EUV loops, since the projection of the field lines at the bottom of AR 11339 leans toward the east, while the loops toward the west. The loops connecting positive and negative polarities of AR 11341 are best overlaid by NLFFF lines than potential ones. The spatial correspondence between the overall shape of the NLFFF field lines and the EUV loops is much improved as shown in Figure2(d). Therefore, the qualitative comparison between the model magnetic field lines and the observed EUV loops indicates that the NLFFF model provides a more consistent field for full-disk magnetic field reconstruction. In the absence of a more reliable quantitative comparison, it remains the best option even if the qualitative nature is not ideal.

In addition to the above comparison to quantify the degree of disagreement between vector field solutions of the two models in the computational volume that are specified on identical sets of grid points, we use five metrics that compare either local characteristics (e.g., vector magnitudes and directions at each point) or the global energy content in addition to the force and divergence integrals as defined in Schrijver, C. J. and Derosa, M. L. and Metcalf, T. R. et al. (2006). The vector correlation $\left(C_{v e c}\right)$ metric of Equation (5) is also used analogous to the standard correlation coefficient for scalar functions. The second metric, $C_{C S}$ is based on the Cauchy-Schwarz inequality $(|\mathbf{a} \cdot \mathbf{b} \leq| \mathbf{a}|| \mathbf{b} \mid$ for any vector $\mathbf{a}$ and b)

$$
C_{\mathrm{CS}}=\frac{1}{N} \sum_{i} \frac{\mathbf{B}_{\mathbf{i}} \cdot \mathbf{b}_{\mathbf{i}}}{\left|\mathbf{B}_{\mathbf{i}}\right|\left|\mathbf{b}_{\mathbf{i}}\right|}
$$

where $\mathbf{B}=\mathbf{B}_{\text {pot }}, \mathbf{b}=\mathbf{B}_{\mathrm{NLFFF}}$, and $N$ is the number of vectors in the field. This metric is mostly a measure of the angular differences of the vector fields: 


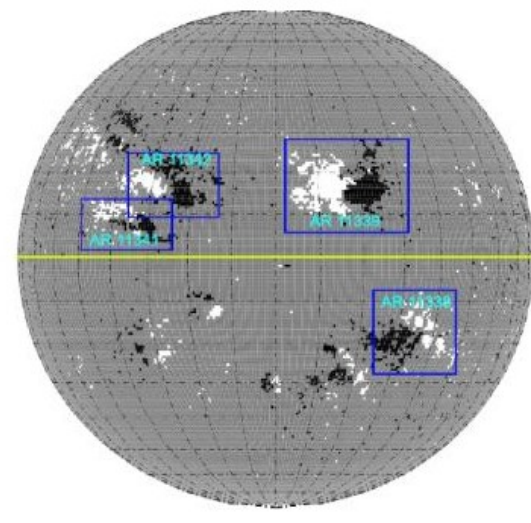

(a) SDO/HMI magnetogram
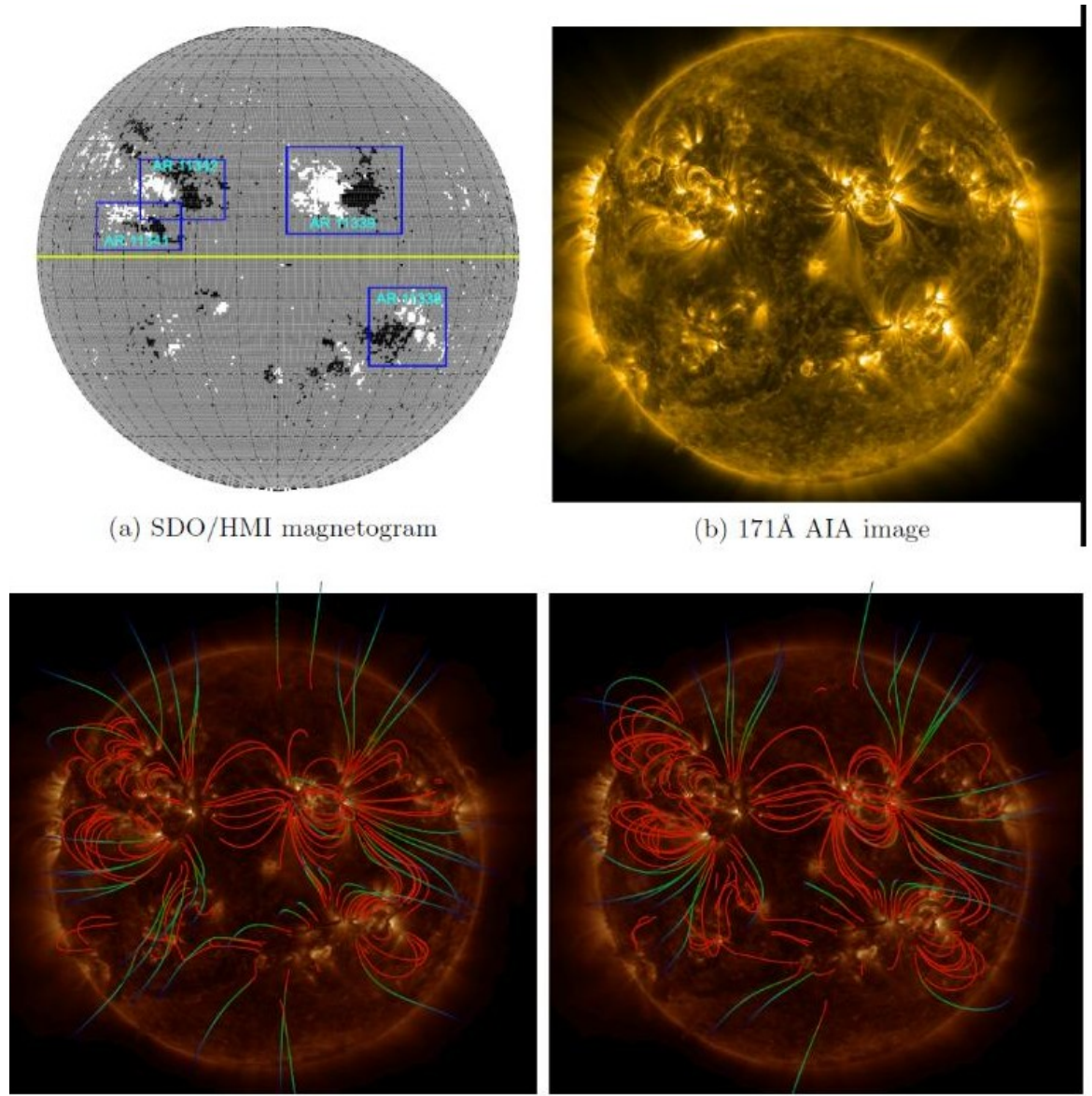

(b) $171 \AA$ AIA image

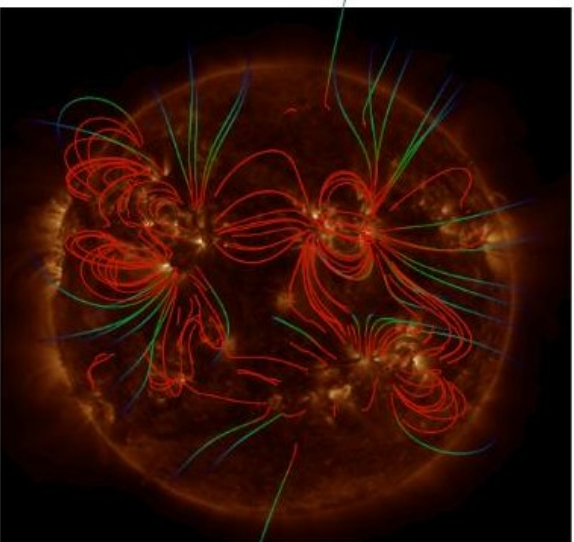

Figure 2. a) Full-disk SDO/HMI magnetogram, the rectangles figure outline the main active regions during this observation and the yellow line indicates the solar equator $\left(\theta=0^{\circ}\right)$, b) Full-disk AIA $171 \AA$ image. Both data sets were obtained on November 09, 2011 around 17:45UT, c) Field lines of the potential field model at 17:45 UT overlaid on AIA $193 \AA$ image and d) Field lines of the NLFFF model at 17:45 UT overlaid on AIA 193A image. Green and red lines represent open and closed magnetic field lines.

$C_{C S}=1$ when $\mathbf{B}$ and $\mathbf{b}$ are parallel and $C_{C S}=-1$ if they are anti-parallel; $C_{C S}=0$ if $\mathbf{B}_{i} \perp \mathbf{b}_{i}$ at each point. Next, we introduce two measures for the vector errors, one normalized to the average vector norm, one averaging over relative differences. The normalized vector error $E_{N}$ is defined as

$$
E_{\mathrm{N}}=\sum_{i}\left|\mathbf{b}_{\mathbf{i}}-\mathbf{B}_{\mathbf{i}}\right| / \sum_{i}\left|\mathbf{B}_{\mathbf{i}}\right|
$$

The mean vector error $E_{M}$ is defined as

$$
E_{\mathrm{M}}=\frac{1}{N} \sum_{i} \frac{\left|\mathbf{b}_{\mathbf{i}}-\mathbf{B}_{\mathbf{i}}\right|}{\left|\mathbf{B}_{\mathbf{i}}\right|}
$$


Table 2. Comparision of figure of merits between potential field and NLFFF models. We have used pherical grids of $225 \times 375 \times 425$.

\begin{tabular}{ccccccccc}
\hline Model & $L_{f}$ & $L_{d}$ & $C_{\mathrm{vec}}$ & $C_{\mathrm{CS}}$ & $1-E_{N}$ & $1-E_{M}$ & $\epsilon$ & Time \\
\hline Potential & 0.000 & 0.002 & 1 & 1 & 0 & 0 & 1 & $30 \mathrm{~min}$ \\
NLFFF & 0.591 & 0.997 & 0.878 & 0.833 & 0.735 & 0.876 & 1.220 & $4 \mathrm{~h}: 39 \mathrm{~min}$ \\
\hline
\end{tabular}

Unlike the first two metrics, perfect disagreement of the two vector fields results in $1-E_{M}=1-E_{N}=0$. As we are also interested in determining how well the models estimate the energy contained in the field, we use the total magnetic energy in NLFFF model field normalized to the total magnetic energy in potential field as a global measure of the quality of the fit:

$$
\epsilon=\frac{\sum_{i}\left|\mathbf{b}_{\mathbf{i}}\right|^{2}}{\sum_{i}\left|\mathbf{B}_{\mathbf{i}}\right|^{2}}
$$

$\epsilon=1$, if there is no difference between the potential field and the nonlinear force-free model solutions. The degree of convergence towards a force-free and divergence-free model solution can be quantified by the integral measures of the Lorentz force and divergence terms in the minimization functional in Equation (??), computed over the entire model volume V. $L_{f}$ and $L_{d}$ of Equation (??) measure how well the force-free and divergence-free conditions are fulfilled, respectively. In Table2, we provide some quantitative measures to rate the quality (figure of merit) of our reconstruction. Column 1 names the corresponding models. Columns $2-3$ show how well the force and solenoidal condition are fulfilled for both models. The next five columns of Table 2 contain different measures which compare our NLFFF solution with potential field. Those figures of merit indicate that there is a clear difference between potential field and NLFFF models solutions for full-disk. The last column shows the computing time on 1 processor. The figures of merit show that the potential field is far away from the true solutions and contains only $81.97 \%$ of the total magnetic energy.

We estimate the free magnetic energy to be the difference between the extrapolated NLFFF and the potential field with the same normal boundary conditions in the photosphere(Régnier and Priest, 2007) Thalmann, Wiegelmann, and Raouafi, 2008). We therefore estimate the upper limit to the free magnetic energy associated with coronal currents of the form

$$
E_{\text {free }}=\frac{1}{8 \pi} \int_{V}\left(B_{n l f f}^{2}-B_{p o t}^{2}\right) r^{2} \sin \theta d r d \theta d \phi,
$$

Our result for the estimation of free-magnetic energy in Table 3 shows that the NLFFF model has $18.83 \%$ more energy than the corresponding potential field model. Therefore, whenever we try to analysis magnetic field configuration and its magnetic field contents in full-disk, it is inaccurate to use potential field model as it undermines the real features of the field in the corona. 
Table 3. The magnetic energy associated with extrapolated potential and NLFFF field configurations from full disk SDO/HMI data.

\begin{tabular}{ccc}
\hline Model & $E_{\text {total }}\left(10^{33} \mathrm{erg}\right)$ & $E_{\text {free }}\left(10^{33} \mathrm{erg}\right)$ \\
\hline Potential & 7.306 & 0 \\
NLFFF & 8.913 & 1.607 \\
\hline
\end{tabular}

In our previous work (Tadesse et al., 2012a), we have studied the connectivity between three neighbouring active regions. In this work, we study the magnetic and electric connectivities between active regions in northern and southern solar hemispheres using full-disk HMI data. During this observation, there were three active regions in the northern hemisphere and one active region with surrounding sunspots in the southern hemisphere. As a result the total unsigned magnetic field flux in the northern hemisphere is larger than that of the southern one. In order to quantify these connectivities, we have calculated the magnetic flux and the electric currents shared between those active regions. For the magnetic flux, e.g., we use

$$
\Phi_{\alpha \beta}=\sum_{i}\left|\mathbf{B}_{i} \cdot \hat{r}\right| R_{\odot}^{2} \sin \left(\theta_{i}\right) \Delta \theta_{i} \Delta \phi_{i}
$$

where the summation is over all pixels of $\mathrm{AR}_{\alpha}$ from which the field line ends in $\mathrm{AR}_{\beta}$ or $i \in \mathrm{AR}_{\alpha} \|$ conjugate footpoint $(i) \in \mathrm{AR}_{\beta}$. The indices $\alpha$ and $\beta$ denote the active region. For the electric current we replace the magnetic field, $\mathbf{B}_{i} \cdot \hat{r}$, by the vertical current density $\mathbf{J}_{i} \cdot \hat{r}$ in Equation (11). Table 4 shows the percentage of the total magnetic flux and electric current shared between the ARs 11339 and 11338 and between AR 11341 and sunspots in the southern hemisphere (see Fig. 3). We have calculated total an unsigned flux for each active regions 11339 and 11341 (both being in northern hemisphere) and the flux due to those field lines ending in the AR 11338 and magnetic patches in the southern hemisphere, respectively. The first column of Table 4 shows that $7.31 \%(6.17 \%)$ of positive/negative polarity of AR 11339 (11341) in the northern hemisphere is connected to positive/negative polarity of AR 11338 (magnetic patches) in southern hemisphere for potential field configuration. The percentage share between those ARs are 7.57\%(6.91\%) for NLFFF configuration. In Table 4 , the percentages in the bracket show the share in electric current between the corresponding active regions and small magnetic patches. Figure 3 display the trans-equatorial loops connecting those active regions. In the figure, we rotate those active regions to the limb to show loops connecting the two hemispheres. The surface contour plots in Figures 4 and 5 show the magnetic flux and electric current density flux of field lines crossing equatorial plane, respectively. From those figures we can see that NLFFF model has more magnetic and electric current density fluxes compared to the potential field. Figure 5 shows that there 
are more electric currents crossing the plane carried by trans-equatorial loops connecting AR 11341 in northern hemisphere to sunspots in the south than those currents connecting ARs 11339 and 11338. This indicate that much of the trans-equatorial loops connecting ARs 11339 and 11338 are potential. In general the two hemispheres are more magnetically connected than electric current. However, one has to use NLFFF model over potential model to study magnetic field structure in any phenomena involving the field.

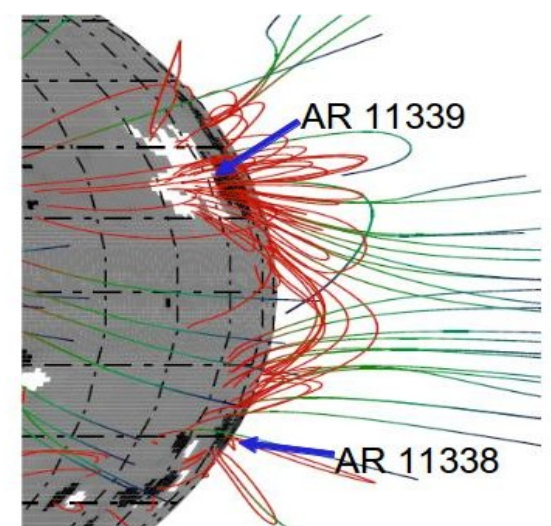

(a) Potential field

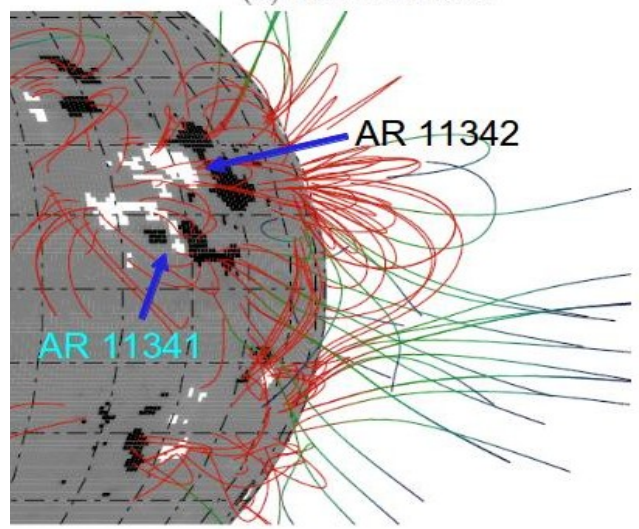

(c) Potential field

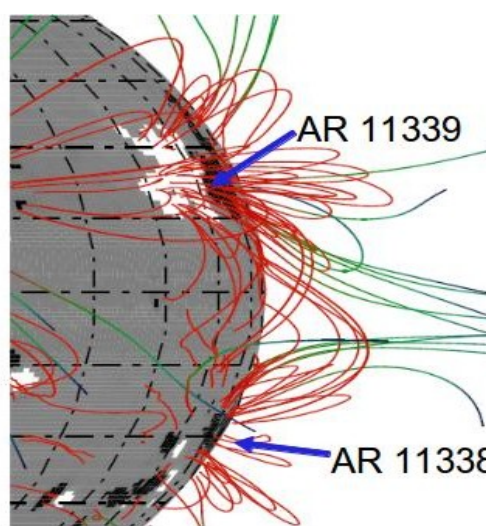

(b) NLFF field

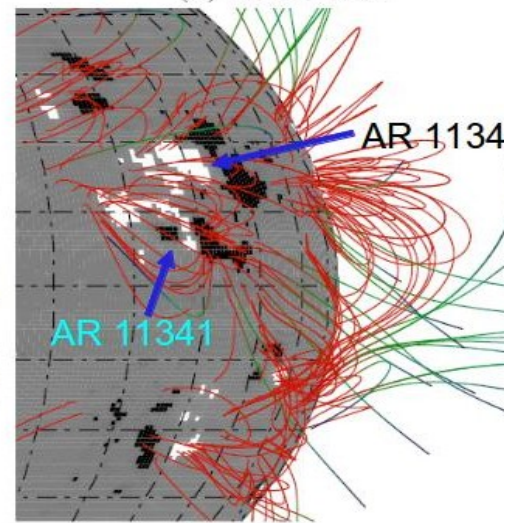

(d) NLFF field

Figure 3. a) Selected potential field lines connecting ARs 11338 and 11339 rotated to limb. b) The corresponding field lines for NLFFF in a), c) Selected potential field lines connecting AR 11341 and Sun spots in southern hemisphere rotated to limb d) The corresponding field lines for NLFFF in c).

\section{Conclusion and outlook}

Both potential and nonlinear force-free field (NLFFF) codes in Cartesian geometry are not well suited for larger domains, since the spherical nature of the 


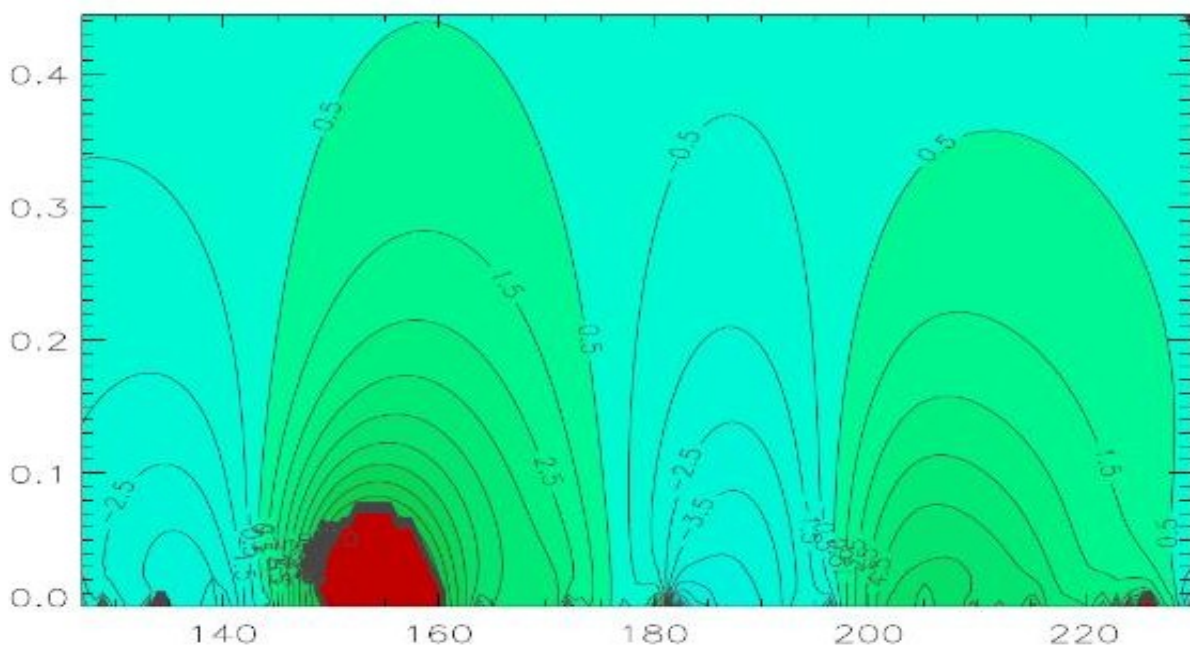

(a) Potential field

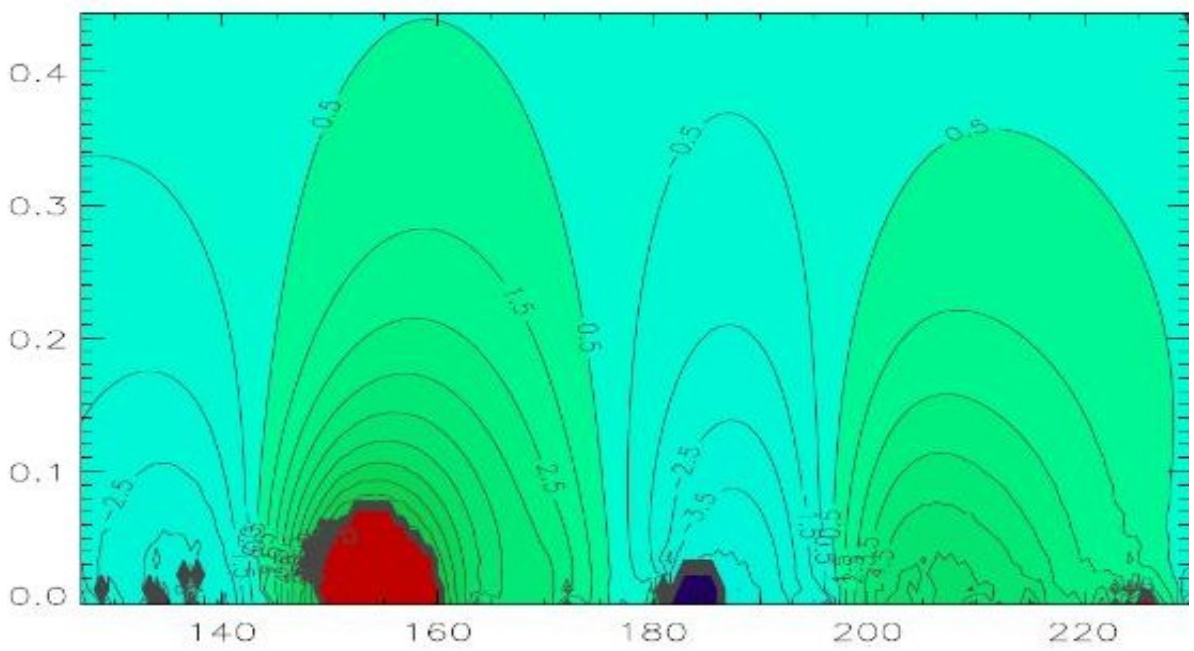

(b) NL.FF field

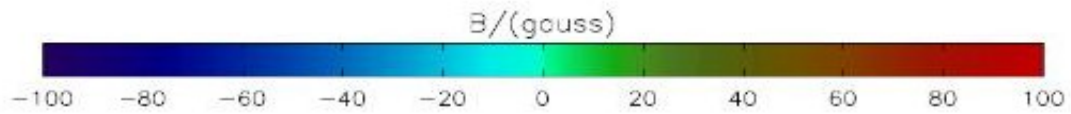

Figure 4. Surface contour plot of perpendicular component of magnetic fields crossing a plane of the solar equator $\left(\theta=0^{\circ}\right)$. The color coding shows $B_{\perp}$ crossing the $\theta=0^{\circ}$ equatorial plane. The vertical and horizontal axes show height, $r$ (in solar radius) and longitude, $\phi$ (in degree) on the plane. 


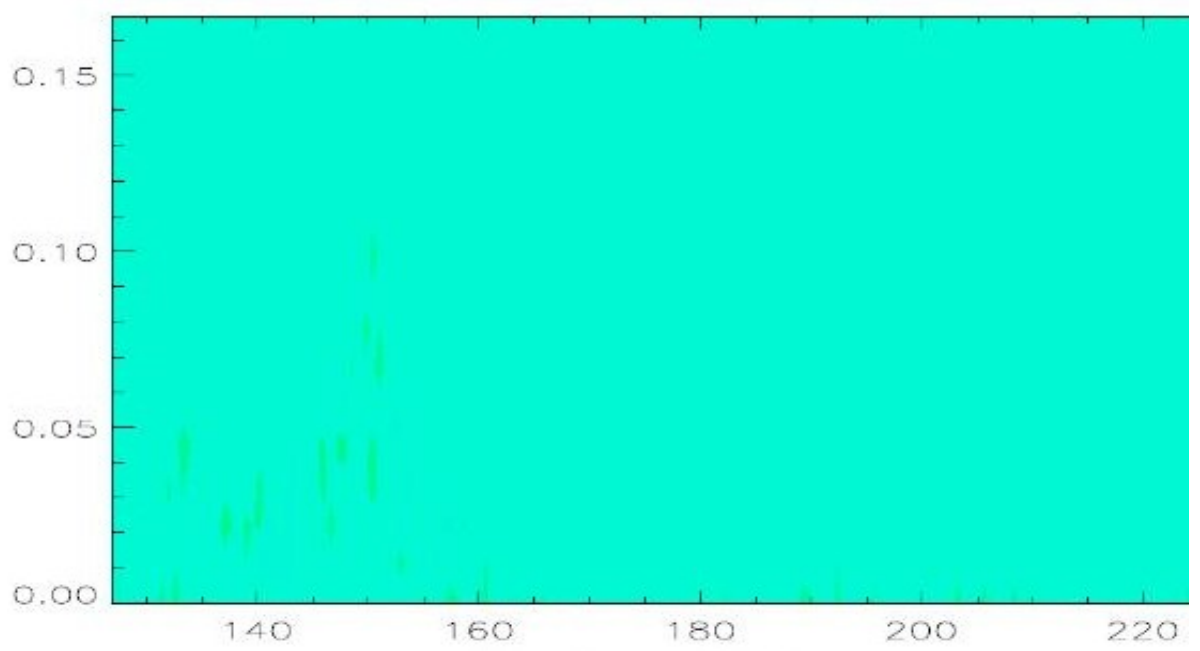

(a) Surface potential field

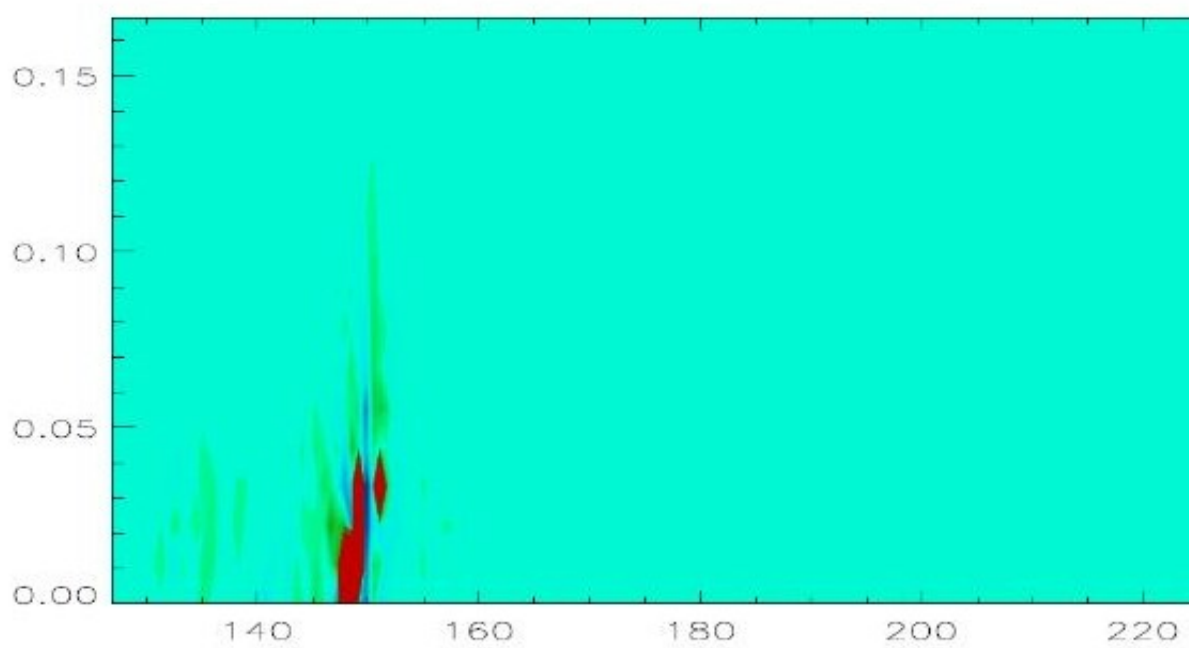

(b) Surface NLFF field

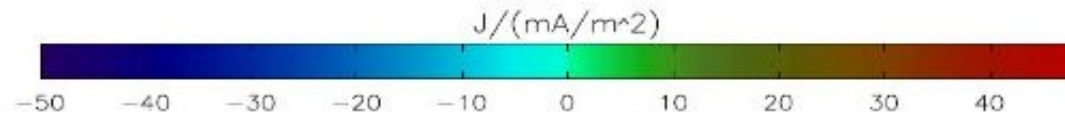

Figure 5. Surface contour plot of perpendicular component of electric current density crossing a plane of the solar equator $\left(\theta=0^{\circ}\right)$. The color coding shows $J_{\perp}$ crossing the $\theta=0^{\circ}$ equatorial plane. The vertical and horizontal axes show height, $r$ (in solar radius) and longitude, $\phi$ (in degree) on the plane. 
Table 4. The percentage of the total magnetic flux and electric current density shared between the ARs 11339 and 11338 and between AR 11341 and sunspots in the southern hemisphere ( see Fig. 3. The percentage in brackets denote that of electric currents.

\begin{tabular}{ccc}
\hline Model & AR $11339 \longmapsto$ AR 11338 & AR $11341 \longmapsto$ Patches in South \\
\hline Potential & $7.31(0.0)$ & $6.17(0.01)$ \\
NLFFF & $7.57(0.13)$ & $6.91(3.35)$ \\
\hline
\end{tabular}

solar surface cannot be neglected when the field of view is large. One of the most significant results of Solar Dynamic Obseratory (SDO) mission to date has been repeated observations of large, almost global scale events in which large scale connection between active regions may play fundamental role. Therefore, it appears prudent to implement a NLFFF procedure in spherical geometry for use when large scale boundary data are available, such as from the Helioseismic and Magnetic Imager (HMI) on board SDO.

In this study, we have investigated the coronal magnetic field associated with full solar disk on November 092011 by analysing SDO/HMI data using potential and NLFFF models. During this particular observation, there were three active regions in the northern hemisphere and one active region surrounded by sunspots in the south. We have used our spherical NLFFF and potential codes to compute the magnetic field solutions in full-disk. For computing the potential field, we use the preprocessed radial component $\mathbf{B}_{r}$ of the HMI-data using spherical harmonic expansion. We implement our NLFFF code initialized by the this potential field solution (except the lower observed bottom boundary) during relaxation towards force-freeness state in the computational volume.

We have compared the magnetic field solutions from both models. The qualitative comparison between the model magnetic field lines and the observed EUV loops indicates that the NLFFF model provides a more consistent field for fulldisk magnetic field reconstruction. In addition to this, the figures of merits have been used to quantify the disagreements between the two models field lines. The figures of merit indicate that the magnetic field lines structures are not potential in full-disk field-of-views. However, much of the trans-equatorial field lines connecting the active regions in the northern and southern hemispheres are potential. This indicates that the two solar hemispheres are more magnetically connected than electric current. The magnetic field lines obtained from nonlinear force-free extrapolation bear larger total magnetic energy that of the potential field model. Therefore, one has to use NLFFF model over potential model to study magnetic field structure in any phenomena involving the field in full-disk.

\section{Acknowledgements}

Data are courtesy of NASA/SDO and the AIA and HMI science teams. SOLIS/VSM vector magnetograms are produced cooperatively by NSF/NSO and 
NASA/LWS. The National Solar Observatory (NSO) is operated by the Association of Universities for Research in Astronomy, Inc., under cooperative agreement with the National Science Foundation. This work was supported by NASA grant NNX07AU64G and the work of T. Wiegelmann was supported by DLR-grant 50 OC 4530501.

\section{References}

Aly, J.J.: 1989,. Sol. Phys. 120, 19.

Amari, T., Aly, J.: 2010,. A\&A 522, A52.

Amari, T., Luciani, J.F., Aly, J.J., Tagger, M.: 1996,. ApJL 466, L39.

Aschwanden, M.J., Malanushenko, A.: 2012,. Sol. Phys., 182. doi 10.1007/s11207-012-0070-1

DeRosa, M. L. and Schrijver, C. J. and Barnes, G. et al.: 2009,. ApJ 696, 1780.

Gary, G.A.: 2001,. Sol. Phys. 203, 71.

Guo, Y., Ding, M.D., Liu, Y., Sun, X.D., DeRosa, M.L., Wiegelmann, T.: 2012,. ApJ 760, 47.

Inhester, B., Wiegelmann, T.: 2006,. Sol. Phys. 235, 201.

Jiang, C., Feng, X.: 2012,. ApJ 749, 135.

Jiang, C., Feng, X., Xiang, C.: 2012,. ApJ 755, 62.

Leka, K.D., Barnes, G., Crouch, t.: 2009,. Sol. Phys. 260, 83.

Lemen, J. R. and Title, A. M. and Akin, D. J. et al.: 2012,. Sol. Phys. 275, 17.

Lin, H., Penn, M.J., Tomczyk, S.: 2000,. ApJL 541, L83.

Liu, Y., Lin, H.: 2008,. ApJ 680, 1496.

Metcalf, T.R.: 1994,. Sol. Phys. 155, 235.

Metcalf, T. R. and De Rosa, M. L. and Schrijver, C. J. et al.: 2008,. Sol. Phys. 247, 269.

Metcalf, T. R. and Leka, K. D. and Barnes, G. et al.: 2006,. Sol. Phys. 237, 267.

Molodensky, M.M.: 1969,. Soviet Astron.-AJ 12, 585.

Régnier, S., Priest, E.R.: 2007,. ApJL 669, L53.

Sakurai, T.: 1989,. Space Sci. Rev. 51, 11.

Schatten, K.H., Wilcox, J.M., Ness, N.F.: 1969,. Sol. Phys. 6, 442.

Schmidt, H.U.: 1964, In: The Physics of Solar Flares, 107.

Schou, J. and Scherrer, P. H. and Bush, R. I. et al.: 2012,. Sol. Phys. 275, 229.

Schrijver, C.J., De Rosa, M.L.: 2003,. Sol. Phys. 212, 165.

Schrijver, C.J., Title, A.M.: 2005,. ApJ 619, 1077.

Schrijver, C. J. and Derosa, M. L. and Metcalf, T. R. et al.: 2006,. Sol. Phys. 235, 161.

Tadesse, T., Wiegelmann, T., Inhester, B.: 2009,. A\&A 508, 421.

Tadesse, T., Wiegelmann, T., Inhester, B., Pevtsov, A.: 2011,. A\&A 527, A30.

Tadesse, T., Wiegelmann, T., Inhester, B., Pevtsov, A.: 2012a,. Sol. Phys. 277, 119.

Tadesse, T., Wiegelmann, T., Inhester, B., Pevtsov, A.: 2012b,. Sol. Phys. 281, 53.

Tadesse, T., Wiegelmann, T., Inhester, B., MacNeice, P., Pevtsov, A., Sun, X.: 2012c, Fulldisk nonlinear force-free field extrapolation of SDO/HMI and SOLIS/VSM magnetograms. ArXiv e-prints.

Thalmann, J.K., Wiegelmann, T., Raouafi, N.E.: 2008,. A\&A 488, L71.

Valori, G., Kliem, B., Keppens, R.: 2005,. A\&A 433, 335.

Wang, Y.M., Sheeley, N.R. Jr.: 1990,. ApJ 365, 372.

Wheatland, M.S.: 2004,. Sol. Phys. 222, 247.

Wheatland, M.S., Leka, K.D.: 2011,. ApJ 728, 112.

Wheatland, M.S., Régnier, S.: 2009,. ApJL 700, L88.

Wheatland, M.S., Sturrock, P.A., Roumeliotis, G.: 2000,. ApJ 540, 1150.

Wiegelmann, T.: 2004,. Sol. Phys. 219, $87-108$.

Wiegelmann, T.: 2007,. Sol. Phys. 240, 227-239.

Wiegelmann, T., Inhester, B.: 2010,. A\&A 516, A107.

Wiegelmann, T., Sakurai, T.: 2012, Living Reviews in Sol. Phys. 9, 5.

Wiegelmann, T., Inhester, B., Sakurai, T.: 2006,. Sol. Phys. 233, 215.

Wiegelmann, T. and Thalmann, J. K. and Inhester, B. and Tadesse, T. et al.: 2012,. Sol. Phys. $\mathbf{2 8 1}, 37$. 